\title{
High Expression of Galectin-3 in Patients with IgG4-Related Disease: A Proteomic Approach
}

\author{
Adeeb Salah, ${ }^{1,2}$ Hajime Yoshifuji, ${ }^{3}$ Shinji Ito, ${ }^{4}$ Koji Kitagori, ${ }^{3,5}$ \\ Kaori Kiso, ${ }^{6}$ Norishige Yamada, ${ }^{6}$ Toshiki Nakajima, ${ }^{3}$ Hironori Haga, ${ }^{1}$ \\ Tatsuaki Tsuruyama, ${ }^{6}$ and Aya Miyagawa-Hayashino ${ }^{1,5}$ \\ ${ }^{1}$ Department of Diagnostic Pathology, Kyoto University Hospital, Kyoto, Japan \\ ${ }^{2}$ Department of Pathology, Faculty of Medicine and Health Sciences, University of Science and Technology, Sana'a, Yemen \\ ${ }^{3}$ Department of Rheumatology and Clinical Immunology, Graduate School of Medicine, Kyoto University, Kyoto, Japan \\ ${ }^{4}$ Bio Frontier Platform, Graduate School of Medicine, Kyoto University, Kyoto, Japan \\ ${ }^{5}$ Center for Innovation in Immunoregulative Technology and Therapeutics, Graduate School of Medicine, Kyoto University, \\ Kyoto, Japan \\ ${ }^{6}$ Center for Anatomical, Pathological and Forensic Medical Research, Graduate School of Medicine, Kyoto University, Kyoto, Japan
}

Correspondence should be addressed to Aya Miyagawa-Hayashino; ayam@kuhp.kyoto-u.ac.jp

Received 12 January 2017; Revised 24 April 2017; Accepted 26 April 2017; Published 16 May 2017

Academic Editor: Paul E. Swanson

Copyright (C) 2017 Adeeb Salah et al. This is an open access article distributed under the Creative Commons Attribution License, which permits unrestricted use, distribution, and reproduction in any medium, provided the original work is properly cited.

Objectives. Immunoglobulin G4-related disease (IgG4-RD) is a multiorgan condition manifesting itself in different forms. This study aimed to investigate protein expression profiles and to find the possible biomarker for IgG4-RD by liquid chromatography mass spectrometry (LC-MS) using tissue sections in IgG4-RD patients. Methods. Protein expression profiles in five IgG4-related pancreatitis and three normal pancreatic samples were compared using LC-MS and were validated by quantitative real-time PCR (qRT-PCR), immunoblotting, and immunohistochemistry. ELISA was employed in the serum of 20 patients with systemic IgG4$\mathrm{RD}$ before and during steroid treatment. Results. LC-MS indicated that the levels of 17 proteins were significantly higher and 12 others were significantly lower in IgG4-related pancreatitis patients compared to controls. Among these proteins, galectin-3 levels were 13-fold higher in IgG4-related pancreatitis $(P<0.01)$. These results were confirmed by immunoblotting and qRT-PCR. The average number of galectin-3 + cells in various organs of IgG4-RD patients, including salivary glands, lungs, and lymph nodes, was higher than in controls. Galectin-3 was detectable in macrophages, dendritic cells, and stromal myofibroblast-like cells, but not in lymphocytes by immunofluorescence staining. Serum galectin-3 levels were higher in patients with IgG4-RD compared with healthy donors and remained high during steroid therapy. Conclusion. Galectin-3 was overexpressed in IgG4-RD and the levels were indirectly related to clinical activity.

\section{Introduction}

Immunoglobulin G4-related disease (IgG4-RD) is an autoimmune multiorgan condition, characterized by a dense lymphoplasmacytic infiltration with a high number of IgG4positive plasma cells and high IgG4 levels in serum [1,2]. The disease has an indolent course and may only be detected after complications dependent on masses $[1,2]$. IgG4 is synthesized and secreted by plasma cells as a part of an immuneprotective mechanism and accounts for less than $5 \%$ of the total IgG in the serum of healthy individuals. IgG4 is generally considered as an anti-inflammatory immunoglobulin, because the ability to fix complement and bind activating Fc receptors is limited [3]. Thus, it is unclear if this immunoglobulin links to disease pathogenesis $[2,4]$.

Chronic antigen exposure stimulates IgG4 production, leading to a shift in the IgG4:IgG1 ratio. The mechanism driving this switch still remains unclear. However, Th2 interleukins such as IL-4, IL-5, and IL-13 can mediate the transition from IgG1 to IgG4 $[4,5]$. Th2s in CD4+ T cells and 
regulatory $\mathrm{T}$ cells play a role in the excessive production of IgG4 and stromal fibrosis in IgG4-RD [6-9]. However, more recent studies show an abundance of CD4+ cytotoxic T cells and a paucity of Th2 cells in IgG4-RD [10,11]. About $50 \%$ of IgG4-RD patients have also allergic diseases $[2,12]$ and these conflicting results may come from IgG4-RD with or without a history of atopy [13]. Nevertheless, the etiology and pathogenesis of IgG4-RD are not well known.

Recent advancements in technical procedures and bioinformatic methods improved the sensitivity of mass spectrometry, making proteomic analysis on formalin-fixed paraffin embedded (FFPE) tissues possible [14]. In this paper, we aimed to investigate protein expression profiles in IgG4-RD and to identify possible biomarkers against this condition using liquid chromatography mass spectrometry (LC-MS).

\section{Materials and Methods}

2.1. Liquid Chromatography Mass Spectrometry (LC-MS). Proteins for LC-MS analysis were extracted from FFPE tissue samples (five IgG4-related pancreatitis and three normal pancreas tissue) using the Liquid Tissue MS Protein Prep kit (Expression Pathology Inc, Rockville, MD, USA) [15]. All cases analyzed for LC-MS were the partial resection for pancreas. For normal pancreas tissues, negative surgical margins taken at resection for pancreatic cancer were selected. Briefly, after deparaffinization, three $0.4 \mu \mathrm{m}$ thick tissue sections $(10 \times 10 \mathrm{~mm})$ were dissected using a needle and solubilized in $20 \mathrm{uL}$ of Liquid Tissue buffer and protein digestion was performed with trypsin (Promega Corp, Madison, WI, USA) for $18 \mathrm{~h}$ at $37^{\circ} \mathrm{C}$. Samples were dried and solubilized in $0.1 \%$ formic acid (Wako, Osaka, Japan) and $1 \mu \mathrm{g}$ aliquots for each sample were separated by nanoflow reversed-phase LC (NanoLC-Ultra 2D-Plus, Eksigent, Dublin, CA, USA) equipped with cHiPLC Nanoflex (Eksigent). Eluted peptides were analyzed by a quadrupole-time-of-flight hybrid mass spectrometer (Triple TOF5600+ system, AB SCIEX, Framingham, MA, USA).

2.2. Peptides Identification and Quantification. Mass spectra were searched against the Uniprot/Swissprot human proteomic database (2014-6 release) from the European Bioinformatics Institute using ProteinPilot version $4.5 \beta$ (AB SCIEX). False discovery rates (FDRs) were determined after peptide/protein identification using Proteomic System Performance Evaluation Pipeline provided as a part of ProteinPilot (AB SCIEX). Label-free quantification of peptides was performed using Progenesis QI for Proteomics (Nonlinear Dynamics, Newcastle upon Tyne, UK). Proteins identified by at least two distinct peptides were used for further analysis.

2.3. Immunoblotting. Immunoblot analysis was performed for protein extracts from FFPE tissues using the Qproteome FFPE Tissue kit (QIAGEN, Venlo, Netherlands). The antibodies used were anti-galectin-3 (9C4, Abcam, Cambridge, UK, 1:1000 dilution) and anti-beta-actin (Abcam, 1:1000). Protein bands were visualized with a chemiluminescence substrate (Chemi-Lumi One L, Nacalai Tesque, Kyoto, Japan), and blots were imaged using Ez-Capture MG (Daihan Scientific Co., Ltd., Gangwon-do, South Korea). Bands were analyzed using the CS Analyzer (Atto Corporation, Tokyo, Japan).

2.4. Real-Time Quantitative PCR ( $q R T-P C R)$. Total RNA was extracted from FFPE samples using the NucleoSpin total RNA FFPE kit (Macherey-Nagel, Düren, Germany). cDNA synthesis was performed using the ReverTra Ace qPCR RT kit (TOYOBO, Osaka, Japan). Amplifications were carried out in triplicate on a 96-well plate in a $10 \mu \mathrm{L}$ volume per well using Fast SYBR Green Master Mix (Applied Biosystems, Life Technologies, Carlsbad, CA, USA). These reactions were performed with an Applied Biosystems 7500 Fast real-time PCR machine. Expression was normalized to GAPDH, using the $2^{-\triangle \Delta \mathrm{Ct}}$ method. The primer sequences were LGALS3 (forward: $5^{\prime}$-GCCTCGCATGCTGATAACAA-3', reverse: $5^{\prime}$ CGTGGGTTAAAGTGGAAGGC-3') and GAPDH (forward: $5^{\prime}$-GGTATCGTGGAAGGACTCATGAC-3', reverse: $5^{\prime}$-ATGCCAGTGAGCTTCCCGTTCAGC-3').

2.5. Immunohistochemistry. Analyzed specimens comprised of FFPE tissues from IgG4-RD patients retrieved from the archive of the department of Diagnostic Pathology, Kyoto University Hospital: pancreas $(n=5)$, bile duct $(n=3)$, retroperitoneum $(n=1)$, aorta $(n=1)$, kidney $(n=2)$, salivary gland $(n=6)$, lung $(n=4)$, ureter $(n=1)$, and lymph node $(n=6)$ from 29 patients between January 2007 and August 2015. Paraffin embedded sections from IgG4-RD patients were immunostained with the following antibodies: alpha-smooth muscle actin (1A4, Sigma Aldrich, St. Louis, MO, USA, 1:300), CD3 (F7.2.38, DakoCytomation, Glostrup, Denmark, 1:50), CD11c (5D11, Cell marque, Rocklin, CA, 1:50), CD123 (6H6, eBioscience, San Diego, CA, USA, 1:100), CD20 (Clone L26, mouse monoclonal, DakoCytomation, 1:1), CD68 (PGM1, DakoCytomation, 1:10), galectin-3 (1:100), IgG (EPR4421, Abcam, 1:50), and IgG4 (HP6025, Nichirei Biosciences Inc., Tokyo, Japan, 1:1). The REAL $^{\mathrm{TM}}$ EnVision $^{\mathrm{TM}} / \mathrm{HRP}$ detection system (DakoCytomation, Glostrup, Denmark) was used to detect immunohistochemical signals. Double immunofluorescence staining was performed using the TSA Plus Fluorescence kit (PerkinElmer Inc., Waltham, MA, USA).

2.6. Enzyme-Linked Immunosorbent Assay (ELISA). ELISA was used to measure galectin-3 and IgG4 levels in 37 serum samples from 20 patients with systemic IgG4-RD before and during prednisolone (PSL) treatment (pretreatment sera were available from 11 patients) and from five healthy control individuals (Human galectin-3 Quantikine ELISA Kit, R\&D Systems, Inc, Minneapolis, MN, USA, and Human IgG subclass profile, Novex, Life Technologies, Frederick, MD, USA, resp.). All experiments were performed in duplicate. Results were compared with samples of patients treated with a tapering dose of PSL.

2.7. Patient Consent and Confidentiality. Sample collection and use of clinical records were authorized under the written consent of patients, and the study was conducted according 


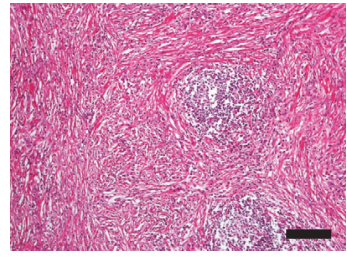

(a)

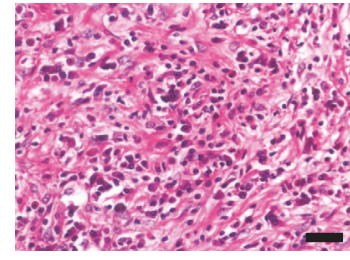

(b)

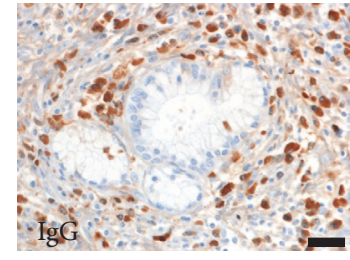

(c)

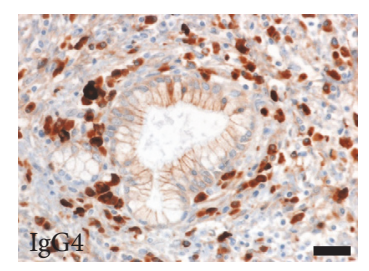

(d)

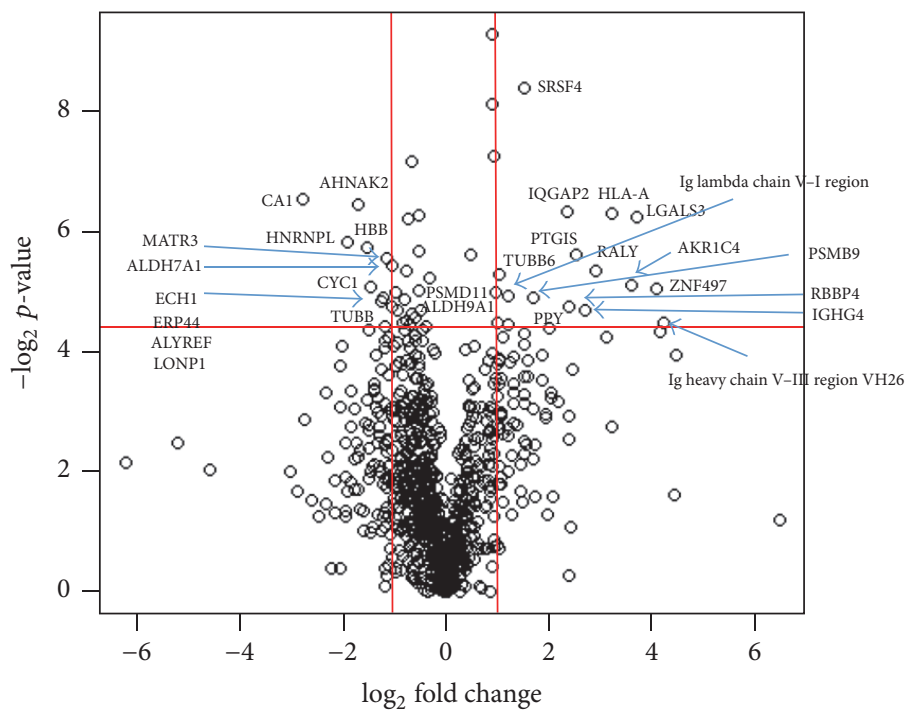

(e)

FIGURE 1: Histologic features of IgG4-related disease (IgG4-RD) and liquid chromatography mass spectrometry (LS-MS) analysis. ((a) to (d)) Pancreatic tissue from patients with IgG4-related pancreatitis. (a) Fibrosis and aggregates of lymphocytes. Hematoxylin and eosin staining, scale bar, $100 \mu \mathrm{m}$. (b) Plasma cell infiltration as one of the histological features of IgG4-RD, Hematoxylin and eosins staining, scale bar, $20 \mu \mathrm{m}$. (c) Immunostaining with anti-IgG antibodies; or (d) with anti-IgG4 antibodies staining. IgG4/IgG > 90\%, scale bar, $20 \mu \mathrm{m}$. IgG4 staining is one of the diagnostic criteria of IgG4-RD. (e) Volcano plot of all differential proteins showing a different abundance in IgG4related pancreatitis $(n=5)$ compared to the healthy pancreas $(n=3)$ samples in our LC-MS analysis. Horizontal axis represents fold change compared to the respective protein in control samples. Vertical axis represents $P$ values. Proteins with a 2 -fold change in their levels and a $P$ value lower that 0.05 were considered significant hits. Fold change values indicate higher $(+)$ or lower $(-)$ expression in IgG4-related pancreatitis samples compared with the controls. Significant proteins are labeled with their gene name in the figure.

to the principles expressed in the Declaration of Helsinki. The Ethics Committee of Kyoto University approved this study (R0305).

2.8. Statistical Analysis. Data were analyzed with GraphPad Prism 6 (mdf, Tokyo, Japan) or R version 3.2.0. To compare continuous variables, unpaired Student's $t$-test or Mann-Whitney $U$ test and two-way analysis of variance (ANOVA) followed by Bonferroni's multiple comparisons test were used. Differences with a $P$ value smaller than 0.05 were considered statistically significant.

\section{Results}

3.1. Protein Identification and Quantification. LC-MS revealed that 956 proteins were differentially expressed between IgG4-related pancreatitis and normal pancreatic samples (Figures 1(a), 1(b), 1(c), and 1(d)). Among these, 352 were significantly upregulated and 604 were downregulated in IgG4-related pancreatitis samples compared with normal pancreatic tissues (Figure 1(e)). Proteins with high confidence values $(P$ value $<0.05)$ and showing a twofold difference in their expression between IgG4-related pancreatitis and normal pancreatic samples were selected for further analysis; the levels of 17 proteins were higher and those of 12 proteins were lower in IgG4-related pancreatitis tissues than in healthy pancreas (Table 1). We selected galectin-3, as it was one of the highly upregulated proteins in IgG4-related pancreatitis (13-fold, $P$ value 0.013 ).

3.2. Validation of Proteomic Data by qRT-PCR, Immunoblotting, and Immunohistochemistry. IgG4-related pancreatitis samples $(n=5)$ showed a 3 -fold up-regulation of LGALS-3 expression compared with healthy pancreas $(n=4$; Figure $2(\mathrm{a})$ ). Immunoblotting showed that galectin-3 protein levels in the same samples as qRT-PCR were 5.1-fold higher in IgG4-related pancreatitis samples than that of normal pancreas (Figures 2(b) and 2(c)).

Immunohistochemical analysis showed that galectin-3 mostly stained stromal spindle cells, macrophages-like cells, 
TABLE 1: Expression profiles of the most significant proteins identified by liquid chromatography mass spectrometry.

\begin{tabular}{|c|c|c|c|c|c|c|}
\hline & Protein name & Gene name & Location & Function & $P$-ANOVA & Fold change \\
\hline 1 & $\begin{array}{l}\text { Serine/arginine-rich splicing } \\
\text { factor } 4\end{array}$ & SRSF4 & Nucleus & $\begin{array}{l}\text { Plays a role in alternative splice site } \\
\text { selection in pre-mRNA splicing }\end{array}$ & 0.003 & 2.84 \\
\hline 2 & Carbonic anhydrase 1 & $C A$ & Cytoplasm & Reversible hydration of carbon dioxide & 0.011 & -6.87 \\
\hline 3 & Protein AHNAK2 & AHNAK2 & Nucleus & $\begin{array}{l}\text { Associate with calcium channel proteins } \\
\text { in cardiomyocytes }\end{array}$ & 0.011 & -3.23 \\
\hline 4 & $\begin{array}{l}\text { Ras GTPase-activating-like } \\
\text { protein IQGAP2 }\end{array}$ & IQGAP2 & Cytoplasm & $\begin{array}{l}\text { Interacts with calmodulin and Rho family } \\
\text { GTPases }\end{array}$ & 0.012 & 5.07 \\
\hline 5 & $\begin{array}{l}\text { HLA class I histocompatibility } \\
\text { antigen, A-74 alpha chain }\end{array}$ & $H L A-A$ & $\begin{array}{l}\text { Plasma } \\
\text { membrane }\end{array}$ & $\begin{array}{l}\text { Involved in the presentation of foreign } \\
\text { antigens to the immune system }\end{array}$ & 0.013 & 9.43 \\
\hline 6 & Galectin-3 & $L G A L S 3$ & $\begin{array}{l}\text { Cytoplasm, } \\
\text { Nucleus }\end{array}$ & $\begin{array}{l}\text { Galactose-specific lectin involved in acute } \\
\text { inflammatory responses }\end{array}$ & 0.013 & 13.01 \\
\hline 7 & $\begin{array}{l}\text { Heterogeneous nuclear } \\
\text { ribonucleoprotein L }\end{array}$ & $H N R N P L$ & $\begin{array}{l}\text { Cytoplasm, } \\
\text { nucleus }\end{array}$ & $\begin{array}{l}\text { Acts as either an activator or repressor of } \\
\text { exon inclusion }\end{array}$ & 0.018 & -3.80 \\
\hline 8 & Hemoglobin subunit beta & $H B B$ & $\begin{array}{l}\text { Red blood } \\
\text { cells }\end{array}$ & $\begin{array}{l}\text { Involved in oxygen transportation from } \\
\text { the lung to peripheral tissues }\end{array}$ & 0.019 & -2.89 \\
\hline 9 & Prostacyclin synthase & PTGIS & $\begin{array}{l}\text { Endoplasmic } \\
\text { reticulum } \\
\text { membrane }\end{array}$ & $\begin{array}{l}\text { Catalyzes the isomerization of } \\
\text { prostaglandin } \mathrm{H} 2 \text { to prostacyclin }\end{array}$ & 0.021 & 5.76 \\
\hline 10 & Matrin-3 & MATR3 & Nucleus & $\begin{array}{l}\text { Role in transcription or may form the } \\
\text { internal fibrogranular network }\end{array}$ & 0.022 & -2.22 \\
\hline 11 & $\begin{array}{l}\text { Alpha-aminoadipic } \\
\text { semialdehyde dehydrogenase }\end{array}$ & $A L D H 7 A 1$ & Cytoplasm & $\begin{array}{l}\text { An important cellular osmolyte and } \\
\text { methyl donor }\end{array}$ & 0.023 & -2.06 \\
\hline 12 & RNA-binding protein Raly & $R A L Y$ & Nucleus & May be involved in pre-mRNA splicing & 0.025 & 7.44 \\
\hline 13 & Tubulin beta- 6 chain & TUBB6 & Cytoplasm & A major constituent of microtubules & 0.026 & 2.04 \\
\hline 14 & $\begin{array}{l}\text { Aldo-keto reductase family } 1 \\
\text { member C4 }\end{array}$ & $A K R 1 C 4$ & Cytoplasm & $\begin{array}{l}\text { Catalyzes the transformation of the } \\
\text { androgen DHT into the less active form }\end{array}$ & 0.030 & 12.08 \\
\hline 15 & $\begin{array}{l}\text { Cytochrome } \mathrm{cl} \text {, heme protein, } \\
\text { mitochondrial }\end{array}$ & CYC1 & Mitochondria & $\begin{array}{l}\text { Transfer electrons to cytochrome } \mathrm{C} \text { in the } \\
\text { mitochondrial respiratory chain }\end{array}$ & 0.030 & -2.77 \\
\hline 16 & Zinc finger protein 497 & ZNF497 & Nucleus & $\begin{array}{c}\text { May be involved in transcriptional } \\
\text { regulation }\end{array}$ & 0.031 & 16.87 \\
\hline 17 & Ig Lambda chainV-I region WAH & NA & Secreted & Antigen binding & 0.033 & 2.29 \\
\hline 18 & Proteasome subunit beta type- 9 & PSMB9 & $\begin{array}{l}\text { Cytoplasm, } \\
\text { Nucleus }\end{array}$ & A multicatalytic proteinase complex & 0.034 & 3.21 \\
\hline 19 & $\begin{array}{c}\text { Delta }(3,5)-\text { Delta } \\
(2,4) \text {-dienoyl-CoA isomerase, } \\
\text { mitochondrial }\end{array}$ & ECH1 & Mitochondria & $\begin{array}{c}\text { Involved in the pathway fatty acid } \\
\text { beta-oxidation }\end{array}$ & 0.034 & -2.31 \\
\hline 20 & $\begin{array}{l}\text { Endoplasmic reticulum resident } \\
\text { protein } 44\end{array}$ & ERP44 & $\begin{array}{l}\text { Endoplasmic } \\
\text { reticulum }\end{array}$ & $\begin{array}{l}\text { Putative role in the control of oxidative } \\
\text { protein folding }\end{array}$ & 0.035 & -2.38 \\
\hline 21 & Histone-binding protein RBBP4 & $R B B P 4$ & Nucleus & $\begin{array}{l}\text { Putative target chromatin assembly } \\
\text { factors }\end{array}$ & 0.037 & 5.21 \\
\hline 22 & THO complex subunit 4 & ALYREF & $\begin{array}{l}\text { Cytoplasm, } \\
\text { nucleus }\end{array}$ & $\begin{array}{l}\text { Export adapter involved in nuclear export } \\
\text { of spliced and un spliced mRNA }\end{array}$ & 0.037 & -2.07 \\
\hline 23 & Ig gamma- 4 chain $\mathrm{C}$ region & IGHG4 & Secreted & Constant region of IgG4 heavy chain & 0.039 & 6.47 \\
\hline 24 & $\begin{array}{l}\text { Ig heavy chain V-III region } \\
\text { VH26 }\end{array}$ & NA & Secreted & Antigen binding & 0.045 & 18.88 \\
\hline 25 & $\begin{array}{l}26 S \text { proteasome non-ATPase } \\
\text { regulatory subunit } 11\end{array}$ & PSMD11 & $\begin{array}{l}\text { Cytoplasm, } \\
\text { nucleus }\end{array}$ & $\begin{array}{l}\text { Involved in the ATP-dependent } \\
\text { degradation of ubiquitinated proteins }\end{array}$ & 0.045 & 2.01 \\
\hline 26 & $\begin{array}{l}\text { 4-trimethylaminobutyraldehyde } \\
\text { dehydrogenase }\end{array}$ & $A L D H 9 A 1$ & Cytoplasm & $\begin{array}{c}\text { Catalyzes the irreversible oxidation of a } \\
\text { broad range of aldehydes }\end{array}$ & 0.046 & 2.28 \\
\hline 27 & $\begin{array}{l}\text { Lon protease homolog, } \\
\text { mitochondrial }\end{array}$ & LONP1 & Mitochondria & $\begin{array}{l}\text { Participates in the regulation of } \\
\text { mitochondrial gene expression }\end{array}$ & 0.047 & -2.26 \\
\hline
\end{tabular}


TABLE 1: Continued.

\begin{tabular}{|c|c|c|c|c|c|c|}
\hline & Protein name & Gene name & Location & Function & $P$-ANOVA & Fold change \\
\hline 28 & Pancreatic prohormone & $P P Y$ & Secreted & $\begin{array}{c}\text { Acts as a regulator of gastrointestinal } \\
\text { functions }\end{array}$ & 0.048 & 4.01 \\
\hline 29 & Tubulin beta chain & $T U B B$ & Cytoplasm & A major constituent of microtubules & 0.049 & -2.80 \\
\hline
\end{tabular}

DHT: dihydrotestosterone; ER: endoplasmic reticulum; NA: not available.

Protein with a 2 -fold change and $P<0.05$ (Mann-Whitney $U$ test followed by Bonferroni's correction) were considered significant. Fold-change values indicate higher $(+)$ or lower $(-)$ expression in IgG4-related pancreatitis samples compared with controls.

Uniprot/Swissprot human proteomic database as reference.

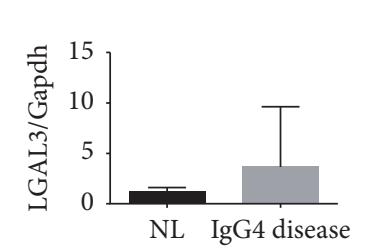

(a)

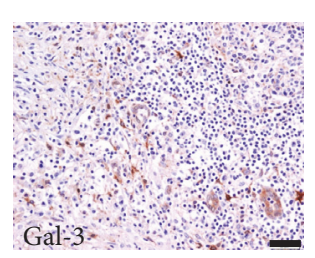

(d)

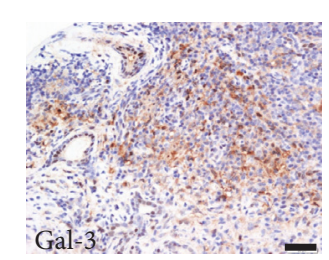

(e)

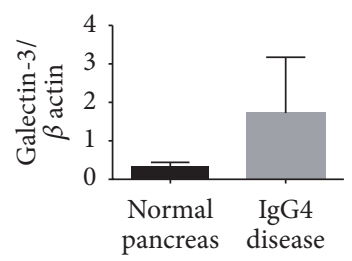

(c)

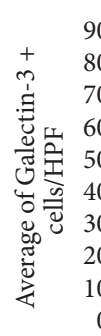

(b)

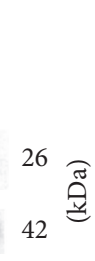

$42 \stackrel{8}{=}$

(1)

FIGURE 2: Validation of galectin-3 expression in patients' tissues. (a) LGALS-3 expression was higher in IgG4-related pancreatitis patients compared to controls. Data are presented as the mean relative expression ratio between the mRNA levels of the LGALS3 gene and the expression of GAPDH. Error bars, standard error of the mean (SEM). (b) Immunoblot analysis showing higher galectin-3 protein levels in IgG4-related pancreatitis samples $(n=5)$ compared with those in the normal pancreas samples $(n=4)$. (c) Quantification of the immunoblot presented in panel (b). Mean band intensity ratio was measured as intensity of galectin-3 band divided by the intensity of the corresponding beta actin band. Bar graphs represent mean values. Error bars, SEM. (d, e) Immunolocalization of galectin-3 in IgG4-RD samples ((d) pancreas; (e) submandibular gland). Note that lymphoid cells in (d) were negative for galectin-3. Scale bar, $20 \mu \mathrm{m}$. (f) Average galectin-3 positive cells in the stroma in different organs in IgG4-RD patients. Positive cells were counted in 3HPF. Numbers in parentheses represent the number of cases. Bar graphs represent mean values. Error bars, SEM.

and epithelial cells, but not mononuclear lymphocyte-like cells (Figures 2(d) and 2(e)).

\subsection{Galectin-3 Is Highly Expressed in Different Organs with} IgG4-RD. To measure the expression of galectin-3 in different organs of IgG4-RD patients, FFPE samples from various organs were stained with anti-galectin-3 antibodies. Since we focused on immune cells, but not on epithelial cells, we counted galectin-3-positive cells in the stroma, including immune and fibroblast-like cells but excluding galectin-3positive epithelial cells, in high-power fields (HPF) from each specimen. The average number of galectin-3-positive stromal cells was higher in organ samples from IgG4-RD patients compared with healthy pancreas or lymph node samples. The differences between healthy pancreas and IgG4-related pancreatitis samples and between healthy lymph node and IgG4 lymphadenopathy were statistically significant $(P$ value $<$ 0.001 for both comparisons) (Figure 2(f)).

3.4. Galectin-3 Is Expressed in Macrophages, Dendritic Cells, and Myofibroblasts. Immunofluorescence staining showed that galectin-3 colocalized with CD68, CD11c, and CD123, 

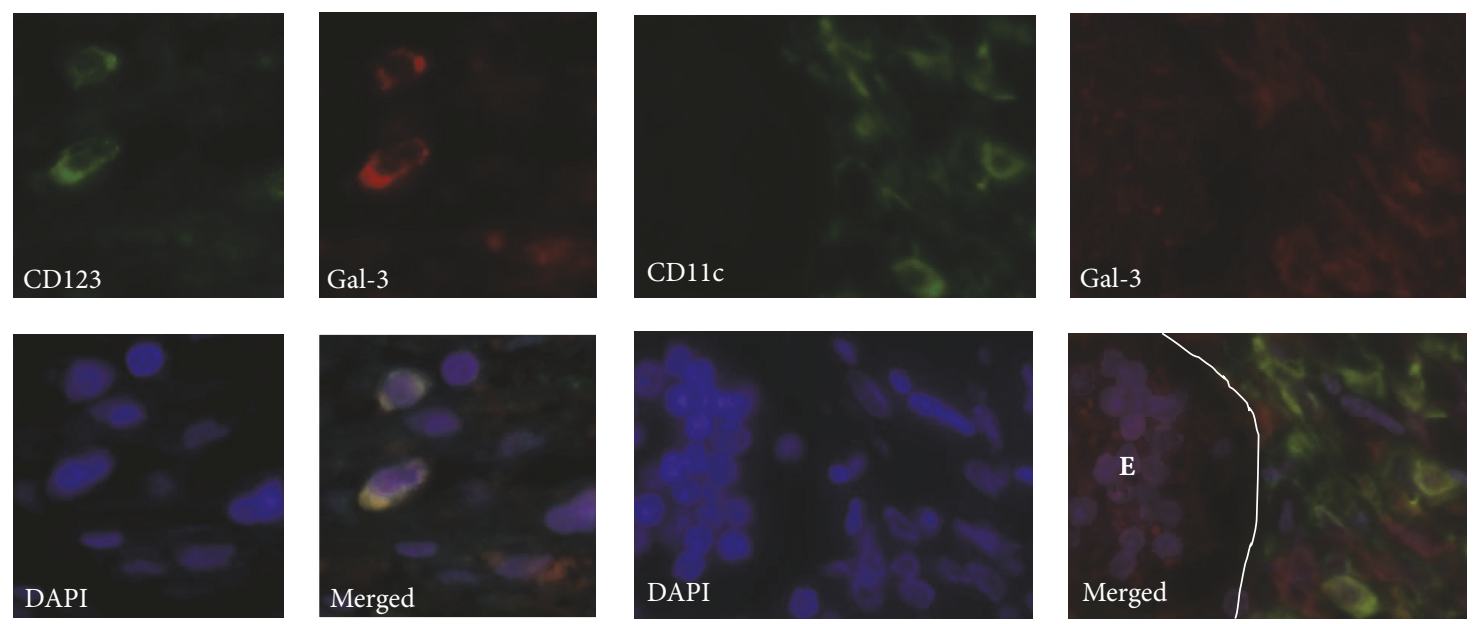

(a)
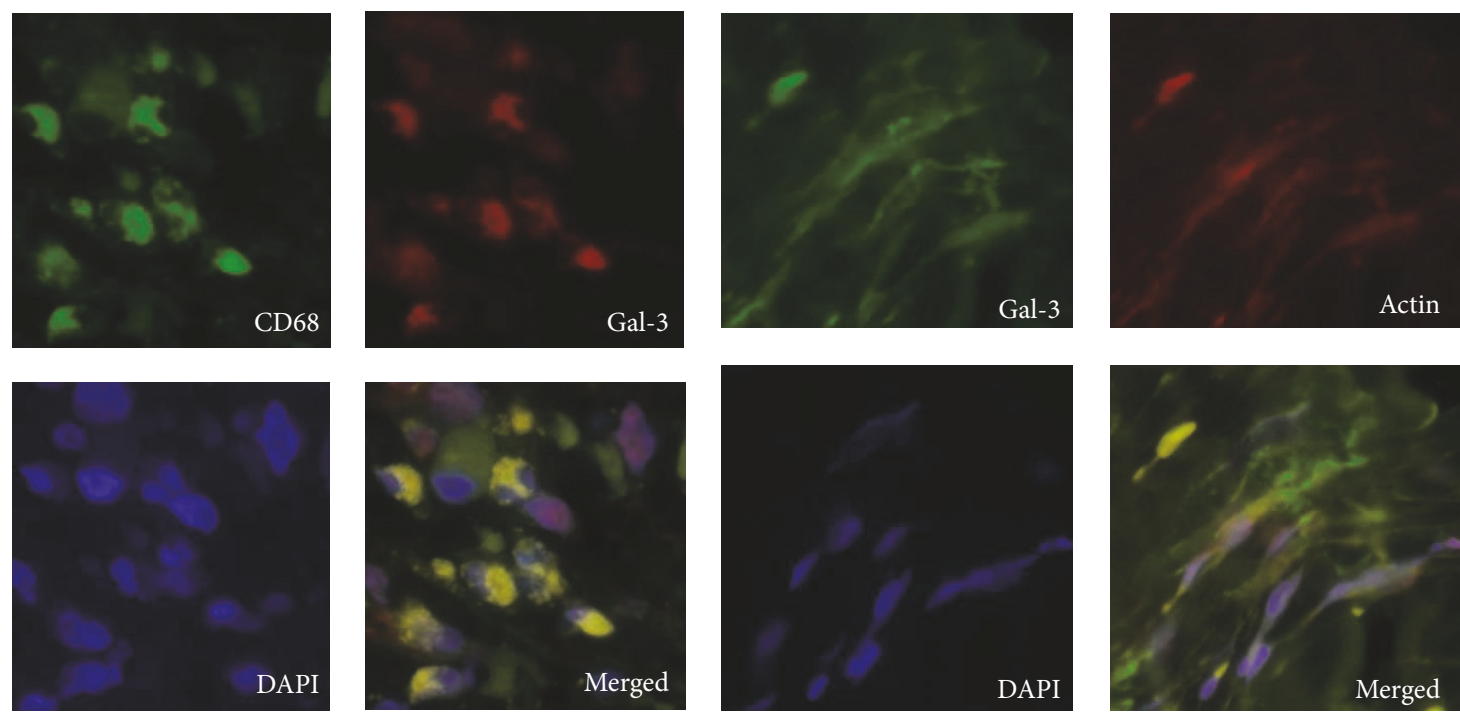

(c)

(d)

FIGURE 3: Immunofluorescent localization galectin-3 in immune and stromal cells of IgG4-RD patients. (a) Immunofluorescent localization of CD123 (green) and galectin 3 (red) in IgG4-RD samples, showing galectin-3 expression in plasmacytoid dendritic cells. (b) Immunofluorescent localization of CD1lc (green) and galectin 3 (red) in IgG4-RD samples, showing galectin-3 expression on myeloid dendritic cells. Note the epithelial cells in the duct, on the left (E), positive for galectin-3. (c) CD68 (green) and galectin-3 (red) in IgG4-RD samples, showing galectin-3 expression on macrophages. (d) Galectin-3 (green) and $\alpha$-smooth muscle actin (red) localization in IgG4-RD samples. Stromal spindle cells were positive for galectin-3, suggesting galectin-3 expression in myofibroblasts. Note the galectin-3 deposition in the stroma. In all experiments, nuclei were stained with DAPI (blue) and images were taken at a magnification of 600x. Gal-3, Galectin-3.

suggesting that galectin-3 was expressed in macrophage, myeloid, and plasmacytoid dendritic cells (Figures 3(a), 3(b), and $3(\mathrm{c}))$. Notably, stromal myofibroblast-like spindle cells expressed both alpha-smooth muscle actin and galectin-3 (Figure 3(d)). Galectin-3 was also visible in the stroma, while we found no galectin-3 staining in CD3-positive $\mathrm{T}$ cells or CD20-positive B cells.

3.5. Galectin-3 Levels in Serum of Patients with Systemic

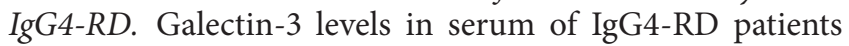
were $7.8 \pm 0.58 \mathrm{ng} / \mathrm{mL}$ and $10.7 \pm 0.58 \mathrm{ng} / \mathrm{mL}$ before and during PSL treatment, respectively, that is, 2 - and 2.5 -fold higher than $3.8 \pm 0.46 \mathrm{ng} / \mathrm{mL}$ found in healthy donors (versus donor $P$ value $<0.01$ for both comparisons; Figure $4(\mathrm{a}))$. We monitored the serum levels of IgG4 and galectin-3 during PSL tapering in five patients for an average of 24 months. While IgG4 serum levels markedly decreased after prednisolone (PSL) therapy, galectin-3 levels in the serum increased. Inversely, lower PSL doses were accompanied by a slight decrease in galectin-3 levels in serum (Figures 4(a) and $4(\mathrm{~b}))$.

Figure 4(c) shows the relative change in galectin-3 levels for five patients before (Galectin-3 levels set to 1) and after treatment (first samples from an average of 1 month after 


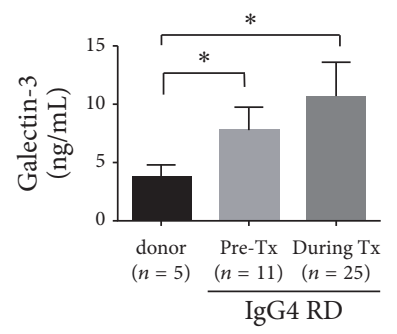

(a)
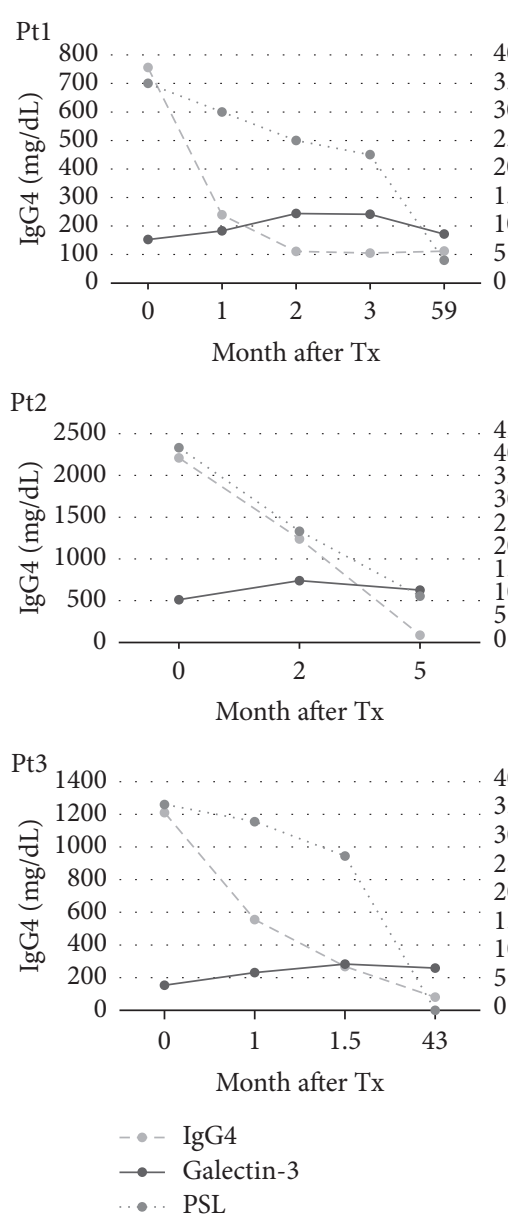

Pt4

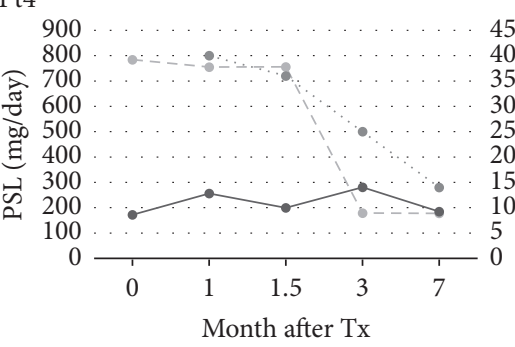

Pt5

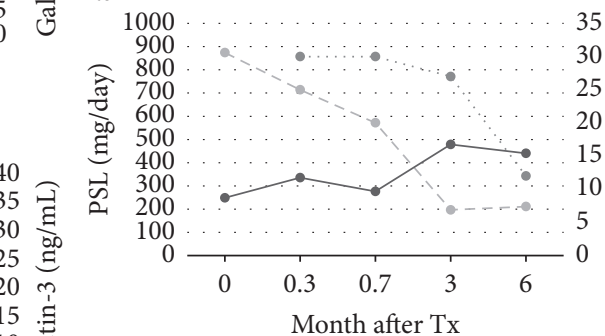

(b)

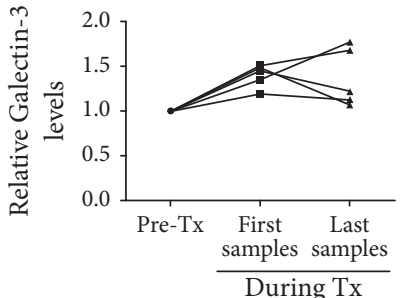

(c)

FIGURE 4: IgG4 and galectin-3 levels in serum after steroid treatment. (a) ELISA assay indicates that galectin-3 levels in serum in patients with IgG4-RD were 2- and 2.5-fold higher, before and after steroid treatment, respectively, compared to the normal control $\left({ }^{*} P<0.05\right)$. Error bars, standard error of the mean (SEM). Tx, treatment. (b) Changes in IgG4 and galectin-3 levels in serum of five IgG4-RD patients before and during steroid treatment. (c) Galectin-3 levels in serum in five steroid-treated IgG4-RD patients. Galectin-3 levels were 1.39-fold higher immediately after the start of the treatment (mean 1 month) and 1.37-fold higher at the last follow-up treatment after 24 months on average, compared to the pretreatment levels.

treatment; range $0.3-2$ months; last samples from an average of 24 months after treatment; range, 5-59 months).

Galectin-3 levels in serum were on average $1.39 \pm 0.058$ fold higher immediately after the start of the PSL therapy (on average 1 month) when compared to galectin-3 levels before the treatment. Galectin-3 levels in serum were on average $1.37 \pm 0.15$-fold higher after the last follow-up treatment, on average 24 months after the start of the therapy, compared to relative to the levels before the treatment (Figure 4(c)).

\section{Discussion}

We chose the protein galectin-3, identified by LC-MS with high confidence and differential expression in FFPE samples of IgG4-related pancreatitis tissues. Galectin-3, encoded by $L G A L S 3$, is a member of the galectin family and expressed in numerous cells, including epithelium cells of gastrointestinal and respiratory tracts and renal tubules, fibroblasts, chondrocytes, osteocytes, and endothelial cells. This protein has 
pro- and antiapoptotic activity [16-18] and regulates human monocyte and macrophage, participating in the autophagy pathway [19-21]. Galectin-3 is also expressed in cells involved in immune responses, such as neutrophils, eosinophils, basophils, mast cells, Langerhans cells, dendritic cells, and monocytes and macrophages [18]. Notably, in resting lymphocytes and several lymphoid cell lines, galectin-3 is not normally expressed, but it can be induced by various stimuli $[18,22,23]$. High galectin-3 expression is observed in many forms of cancer, including thyroid, pancreatic, and colon cancers, and has been linked to cancer progression and metastasis [24, 25].

Galectin-3 is highly expressed in tissues affected by several autoimmune diseases, such as systemic lupus erythematosus, polymyositis, dermatomyositis, rheumatoid arthritis, Behcet's disease, systemic sclerosis, and Crohn's disease. Moreover, galectin-3 shows higher expression in active than in inactive diseases [26-28]. Galectin-3 participates in the development of the T helper-2 (Th2) response [29]. However, the expression and the role of galectin-3 in IgG4-RD remains unknown.

This study demonstrated an increase in galectin-3 expression in IgG4-RD patients. Galectin-3 was expressed in cells involved in immune response activity, including macrophages, dendritic cells, myofibroblasts, and epithelial cells. Galectin-3 signal was found in stromal deposition. Dendritic cells are specialized in antigen presentation cells and connect the innate and adaptive immune system [30]. They have the distinctive ability to stimulate and polarize naive $\mathrm{T}$ cells. For example, the expressions of IL-12 and IL-10 stimulate T helper-1 (Th1) and Th2 activation, respectively [30]. Th2 response controls the production of IgG4 [18]. Plasmacytoid dendritic cells participate in autoimmune diseases, such as IgG4-related pancreatitis, lupus erythematosus, psoriasis, and contact dermatitis, likely contributing to IFN$\alpha$ production $[31,32]$. Galectin-3 secreted by dendritic cells mediates the binding of these cells to lymphocytes and might play a role in cell-cell interactions [33, 34]. Galectin3 binds integrins, the main membrane molecules involved in cell adhesion, including those present on the surface of macrophages such as $\alpha 1 \beta 1$ integrins and the $\alpha$ subunit of $\alpha \mathrm{M} \beta 1$ integrin (CD11b/18, Mac- $1 \mathrm{Ag})$, and these integrins are used as receptors [18, 21]. Galectin-3 is also essential for effective phagocytosis by macrophages to remove apoptotic cells and therefore preventing autoimmune reactions [35]. In addition, endogenous galectin- 3 has been found to drive a Th2 response in both dendritic cells and $\mathrm{T}$ cells, while galectin3 deficiency resulted in the development of a Th1 response [29]. Recent studies show clonally expanded CD4+ cytotoxic $\mathrm{T}$ cells that express granzyme $\mathrm{B}$, perforin, IFN-gamma, and TGF-betal are prominent in IgG4RD $[10,11]$ Rituximabmediated $\mathrm{B}$ cell depletion is associated with a reduction in numbers of disease-associated CD4+ cytotoxic T cells, suggesting that $\mathrm{B}$ cells play an important role in the maintenance of disease-associated $\mathrm{T}$ cell clone [10]. Extracellular galectin-3 directly induces $\mathrm{T}$ cell apoptosis in a carbohydrate dependent manner by binding to its cell surface receptors, CD7 and CD29 [36]. Inhibition of galectin-3 in vivo skewed the balance toward plasma cell differentiation and increased immunoglobulin production through the up-regulation of Blimp-1, a transcription factor responsible for B cell apoptosis and essential for plasma cell commitment, in Trypanosoma cruzi infection model [23]. Additionally, galectin-3 acts as a negative regulator of the differentiation of B-1 lymphocytes into plasma cells [37]. Likewise, IL-4-mediated B cells activation, resulting in their differentiation into memory cells, is accompanied by a significant increase in galectin- 3 expression [23]. Therefore, galectin-3 may act as a protective factor against the progression of IgG4-RD since galectin-3 blocks plasma cell differentiation in B cells and apoptosis of $\mathrm{T}$ cells $[23,36,37]$, although this protein has other biological roles [18].

In our study, galectin-3 serum levels in patients with IgG4-RD before and during PSL treatment were significantly higher compared with those in healthy donors. IgG4 levels in serum decreased dramatically after PSL treatment, while galectin-3 levels in serum remained high throughout the treatment. Galectin-3 serum levels increased with PSL treatment and gradually decreased with the tapering of PSL. This implies that galectin-3 cannot serve as a realistic biomarker for IgG4-RD in routine clinical practice. Glucocorticoid therapy could be a bias, because galectin-3 expression increases significantly in macrophages and lung Clara cells after glucocorticoid treatment [38]. Similarly, mineralocorticoid hormones increase galectin-3 expression in vascular smooth muscle cells [39]. Therefore, the induction of galectin-3 expression after treatment with PSL, a synthetic glucocorticoid, may be in agreement with these previous reports. Galectin-3 expression is higher in SLE and systemic sclerosis patients with active diseases than those with an inactive one. However, galectin-3 expression is not directly correlated with the disease activity and severity indexes in each SLE and systemic sclerosis patient, nor with the duration of the disease [28]. In our study, galectin-3 was present in the stroma and myofibroblasts in IgG4-RD patients. Immune cells subside immediately after steroid therapy, while tissue fibrosis may remain even after treatment [40]. It is difficult to assist tissue fibrosis because follow-up biopsy is uncommon after the treatment of IgG4-RD. The only available data come from a recent work by Della-Torre et al. [41], who found a reduction in the total number of myofibroblasts after rituximab treatment in one patient with cutaneous IgG4-RD. The presence of extracellular galectin-3 can be a determining factor for prolonged galectin-3 levels in serum after PSL treatment. It is possible that galectin-3 seen in patients with IgG4-RD represents the response against inflammation. To understand the significance of galectin-3 in relation to the pathogenesis of IgG4-RD, the decreased expression of galectin-3 in the affected tissues after successful corticosteroid therapy would be important; however no tissues after therapy were available.

In conclusion, galectin-3 was overexpressed in IgG4-RD and the levels were indirectly related to clinical activity and treatment. Further studies will need to explore the exact mechanism behind the function of galectin-3 in IgG4-RD and other autoimmune diseases. 


\section{Disclosure}

Recent address for Dr. Miyagawa-Hayashino is Department of Clinical Pathology, Kansai Medical University, 2-3-1 Shinmachi, Hirakata city, Osaka 573-1191, Japan. Publication of this article was approved by an intellectual property committee composed of representatives from Kyoto University and Astellas Pharma.

\section{Conflicts of Interest}

The authors declare no conflicts of interest. Astellas Pharma had no role in the design of the study, in the collection, analysis, or interpretation of the data, in the writing the manuscript, or in the decision to submit the manuscript for publication.

\section{Acknowledgments}

This work was supported by Astellas Pharma Inc. within the Creation of Innovation Centers for Advanced Interdisciplinary Research Areas Program. The authors thank Ms. Aki Matsumoto, Ms. Chikako Endo, and Mr. Tsugumitsu Kandou for their technical assistance.

\section{References}

[1] T. Kamisawa, N. Funata, Y. Hayashi et al., "A new clinicopathological entity of IgG4-related autoimmune disease," Journal of Gastroenterology, vol. 38, no. 10, pp. 982-984, 2003.

[2] J. H. Stone, Y. Zen, and V. Deshpande, "Mechanisms of disease: IgG4-related disease," The New England Journal of Medicine, vol. 366, no. 6, pp. 539-551, 2012.

[3] M. V. D. N. Kolfschoten, J. Schuurman, M. Losen et al., "Antiinflammatory activity of human IgG4 antibodies by dynamic Fab arm exchange," Science, vol. 317, no. 5844, pp. 1554-1557, 2007.

[4] R. C. Aalberse, S. O. Stapel, J. Schuurman, and T. Rispens, "Immunoglobulin G4: an odd antibody," Clinical and Experimental Allergy, vol. 39, no. 4, pp. 469-477, 2009.

[5] J. Punnonen, G. Aversa, B. G. Cocks et al., "Interleukin 13 induces interleukin 4-independent IgG4 and IgE synthesis and CD23 expression by human B cells," Proceedings of the National Academy of Sciences of the United States of America, vol. 90, no. 8, pp. 3730-3734, 1993.

[6] K. Okazaki, K. Uchida, M. Ohana et al., "Autoimmune-related pancreatitis is associated with autoantibodies and a Th1/Th2type cellular immune response," Gastroenterology, vol. 118, no. 3, pp. 573-581, 2000.

[7] Y. Zen, T. Fujii, K. Harada et al., “Th2 and regulatory immune reactions are increased in immunoglobin G4-related sclerosing pancreatitis and cholangitis," Hepatology, vol. 45, no. 6, pp. 1538-1546, 2007.

[8] H. Kanari, S.-I. Kagami, D. Kashiwakuma et al., "Role of Th2 cells in IgG4-related lacrimal gland enlargement," International Archives of Allergy and Immunology, vol. 152, no. 1, pp. 47-53, 2010.

[9] A. Tanaka, M. Moriyama, H. Nakashima et al., "Th2 and regulatory immune reactions contribute to IgG4 production and the initiation of Mikulicz disease," Arthritis and Rheumatism, vol. 64, no. 1, pp. 254-263, 2012.

[10] H. Mattoo, V. S. Mahajan, T. Maehara et al., "Clonal expansion of CD4(+) cytotoxic T lymphocytes in patients with IgG4related disease," Journal of Allergy and Clinical Immunology, vol. 138, no. 3, pp. 825-838, 2016.

[11] T. Maehara, H. Mattoo, M. Ohta et al., "Lesional CD4+ IFN$\gamma+$ cytotoxic T lymphocytes in IgG4-related dacryoadenitis and sialoadenitis," Annals of the Rheumatic Diseases, 2016.

[12] T. Kamisawa, H. Anjiki, N. Egawa, and N. Kubota, "Allergic manifestations in autoimmune pancreatitis," European Journal of Gastroenterology and Hepatology, vol. 21, no. 10, pp.1136-1139, 2009.

[13] H. Mattoo, E. Della-Torre, V. S. Mahajan, J. H. Stone, and S. Pillai, "Circulating Th2 memory cells in IgG4-related disease are restricted to a defined subset of subjects with atopy," Allergy: European Journal of Allergy and Clinical Immunology, vol. 69, no. 3, pp. 399-402, 2014.

[14] D. C. Vincenti and G. I. Murray, "The proteomics of formalinfixed wax-embedded tissue," Clinical Biochemistry, vol. 46, no. 6, pp. 546-551, 2013.

[15] Y. Kakimoto, S. Ito, H. Abiru et al., "Sorbin and SH3 domaincontaining protein 2 is released from infarcted heart in the very early phase: proteomic analysis of cardiac tissues from patients," Journal of the American Heart Association, vol. 2, no. 6, article e000565, 2013.

[16] F.-T. Liu, R. J. Patterson, and J. L. Wang, "Intracellular functions of galectins," Biochimica et Biophysica Acta, vol. 1572, no. 2-3, pp. 263-273, 2002.

[17] L. Chiariotti, P. Salvatore, R. Frunzio, and C. B. Bruni, "Galectin genes: regulation of expression," Glycoconjugate Journal, vol. 19, no. 7-9, pp. 441-449, 2002.

[18] J. Dumic, S. Dabelic, and M. Flögel, "Galectin-3: an open-ended story," Biochimica et Biophysica Acta, vol. 1760, no. 4, pp. 616635, 2006.

[19] R.-Y. Yang, D. K. Hsu, and F.-T. Liu, "Expression of galectin3 modulates T-cell growth and apoptosis," Proceedings of the National Academy of Sciences of the United States of America, vol. 93, no. 13, pp. 6737-6742, 1996.

[20] S. Sato and R. C. Hughes, "Regulation of secretion and surface expression of Mac-2, a galactoside-binding protein of macrophages," The Journal of Biological Chemistry, vol. 269, pp. 4424-4430, 1994.

[21] B. J. Cherayil, S. J. Weiner, and S. Pillai, "The Mac-2 antigen is a galactose-specific lectin that binds IgE," Journal of Experimental Medicine, vol. 170, no. 6, pp. 1959-1972, 1989.

[22] H. G. Joo, P. S. Goedegebuure, N. Sadanaga, M. Nagoshi, W. Von Bernstorff, and T. J. Eberlein, "Expression and function of galectin-3, a beta-galactoside-binding protein in activated $\mathrm{T}$ lymphocytes," Journal of Leukocyte Biology, vol. 69, no. 4, pp. 555-564, 2001.

[23] E. V. Acosta-Rodriguez, C. L. Montes, C. C. Motran et al., "Galectin-3 mediates IL-4-induced survival and differentiation of B cells: functional cross-talk and implications during trypanosoma cruzi infection," The Journal of Immunology, vol. 172, no. 1, pp. 493-502, 2004.

[24] H. A. Idikio, "Galectin-3 and beclin1/Atg6 genes in human cancers: using cDNA tissue panel, qRT-PCR, and logistic regression model to identify cancer cell biomarkers," PLOS ONE, vol. 6, no. 10, article e26150, 2011.

[25] S. Nakahara, N. Oka, and A. Raz, "On the role of galectin-3 in cancer apoptosis," Apoptosis, vol. 10, no. 2, pp. 267-275, 2005. 
[26] Y. Lim, D.-Y. Lee, S. Lee et al., "Identification of autoantibodies associated with systemic lupus erythematosus," Biochemical and Biophysical Research Communications, vol. 295, no. 1, pp. 119124, 2002.

[27] E. Jensen-Jarolim, C. Neumann, G. Oberhuber et al., "AntiGalectin-3 IgG autoantibodies in patients with Crohn's disease characterized by means of phage display peptide libraries," Journal of Clinical Immunology, vol. 21, no. 5, pp. 348-356, 2001.

[28] S. S. Koca, F. Akbas, M. Ozgen et al., "Serum galectin-3 level in systemic sclerosis," Clinical Rheumatology, vol. 33, no. 2, pp. 215-220, 2014.

[29] J. Saegusa, D. K. Hsu, H.-Y. Chen et al., "Galectin-3 is critical for the development of the allergic inflammatory response in a mouse model of atopic dermatitis," American Journal of Pathology, vol. 174, no. 3, pp. 922-931, 2009.

[30] A. Savina and S. Amigorena, "Phagocytosis and antigen presentation in dendritic cells," Immunological Reviews, vol. 219, no. 1, pp. 143-156, 2007.

[31] Y. Arai, K. Yamashita, K. Kuriyama et al., "Plasmacytoid dendritic cell activation and IFN- $\alpha$ production are prominent features of murine autoimmune pancreatitis and human IgG4related autoimmune pancreatitis," Journal of Immunology, vol. 195, no. 7, pp. 3033-3044, 2015.

[32] A. Wollenberg, M. Wagner, S. Günther et al., "Plasmacytoid dendritic cells: a new cutaneous dendritic cell subset with distinct role in inflammatory skin diseases," Journal of Investigative Dermatology, vol. 119, no. 5, pp. 1096-1102, 2002.

[33] C. Théry, M. Boussac, P. Véron et al., "Proteomic analysis of dendritic cell-derived exosomes: a secreted subcellular compartment distinct from apoptotic vesicles," The Journal of Immunology, vol. 166, no. 12, pp. 7309-7318, 2001.

[34] V. V. R. Swarte, R. E. Mebius, D. H. Joziasse, D. H. Van Den Eijnden, and G. Kraal, "Lymphocyte triggering via L-selectin leads to enhanced galectin-3-mediated binding to dendritic cells," European Journal of Immunology, vol. 28, no. 9, pp. 2864-2871, 1998.

[35] H. Sano, D. K. Hsu, J. R. Apgar et al., "Critical role of galectin-3 in phagocytosis by macrophages," The Journal of Clinical Investigation, vol. 112, no. 3, pp. 389-397, 2003.

[36] T. Fukumori, Y. Takenaka, T. Yoshii et al., "CD29 and CD7 mediate galectin-3-induced type II T-cell apoptosis," Cancer Research, vol. 63, pp. 8302-8311, 2003.

[37] F. L. Oliveira, R. Chammas, L. Ricon et al., "Galectin-3 regulates peritoneal B1-cell differentiation into plasma cells," Glycobiology, vol. 19, no. 11, pp. 1248-1258, 2009.

[38] C. A. Maldonado, V. Sundblad, M. Salatino et al., "Cell-type specific regulation of galectin-3 expression by glucocorticoids in lung Clara cells and macrophages," Histology and Histopathology, vol. 26, no. 6, pp. 747-759, 2011.

[39] L. Calvier, M. Miana, P. Reboul et al., "Galectin-3 mediates aldosterone-induced vascular fibrosis," Arteriosclerosis, Thrombosis, and Vascular Biology, vol. 33, no. 1, pp. 67-75, 2013.

[40] I. Mizushima, K. Yamada, H. Fujii et al., "Clinical and histological changes associated with corticosteroid therapy in IgG4related tubulointerstitial nephritis," Modern Rheumatology, vol. 22, no. 6, pp. 859-870, 2012.

[41] E. Della-Torre, E. Feeney, V. Deshpande et al., "B-cell depletion attenuates serological biomarkers of fibrosis and myofibroblast activation in IgG4-related disease," Annals of the Rheumatic Diseases, vol. 74, no. 12, pp. 2236-2243, 2015. 


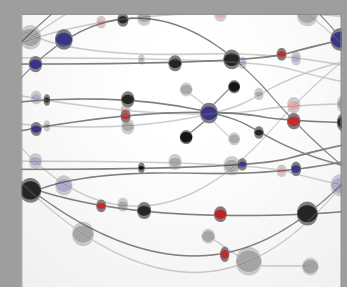

The Scientific World Journal
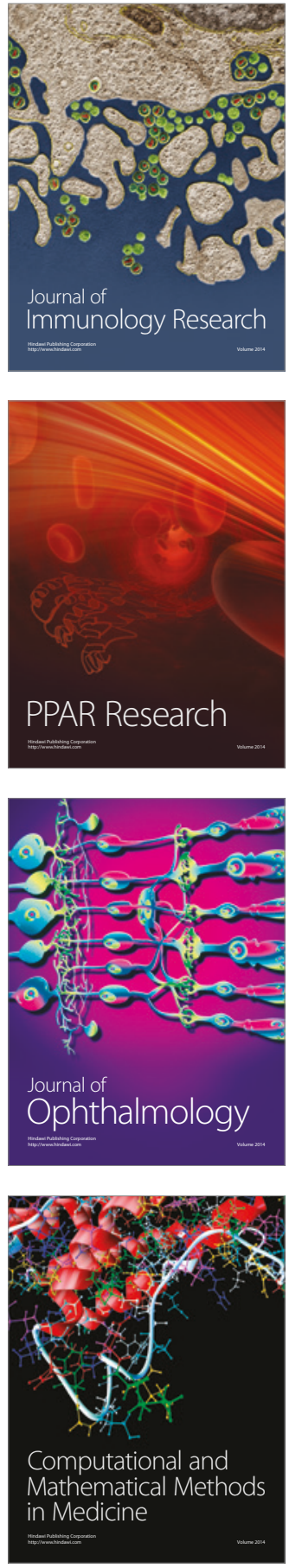

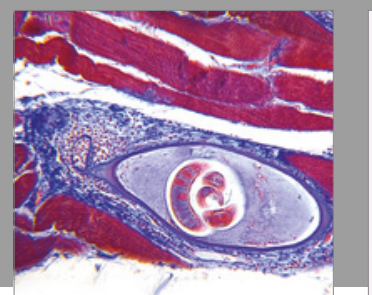

Gastroenterology Research and Practice
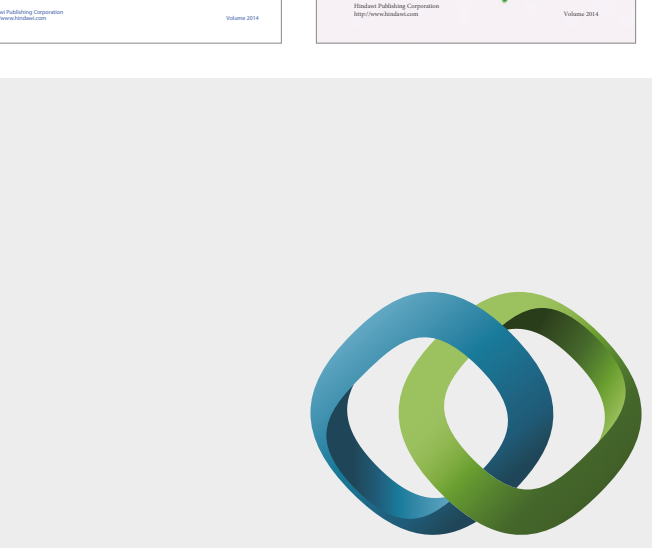

\section{Hindawi}

Submit your manuscripts at

https://www.hindawi.com
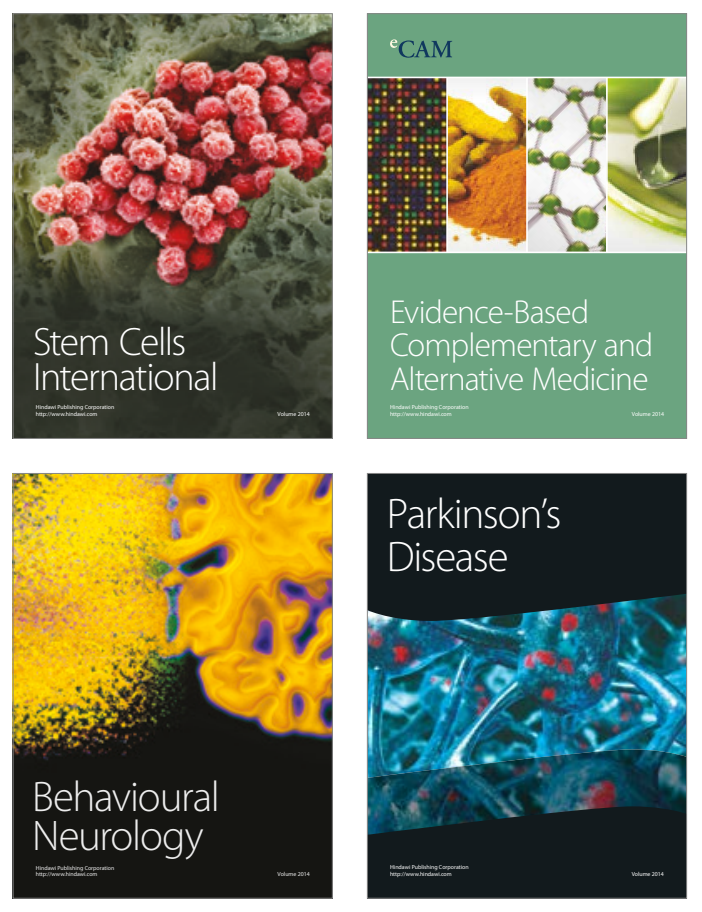
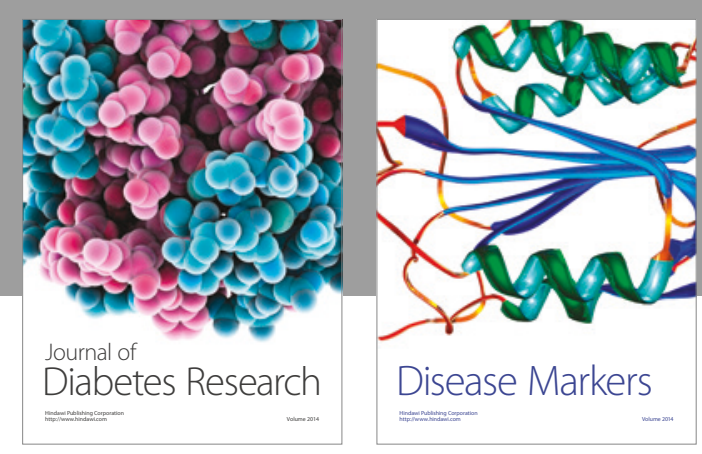

Disease Markers
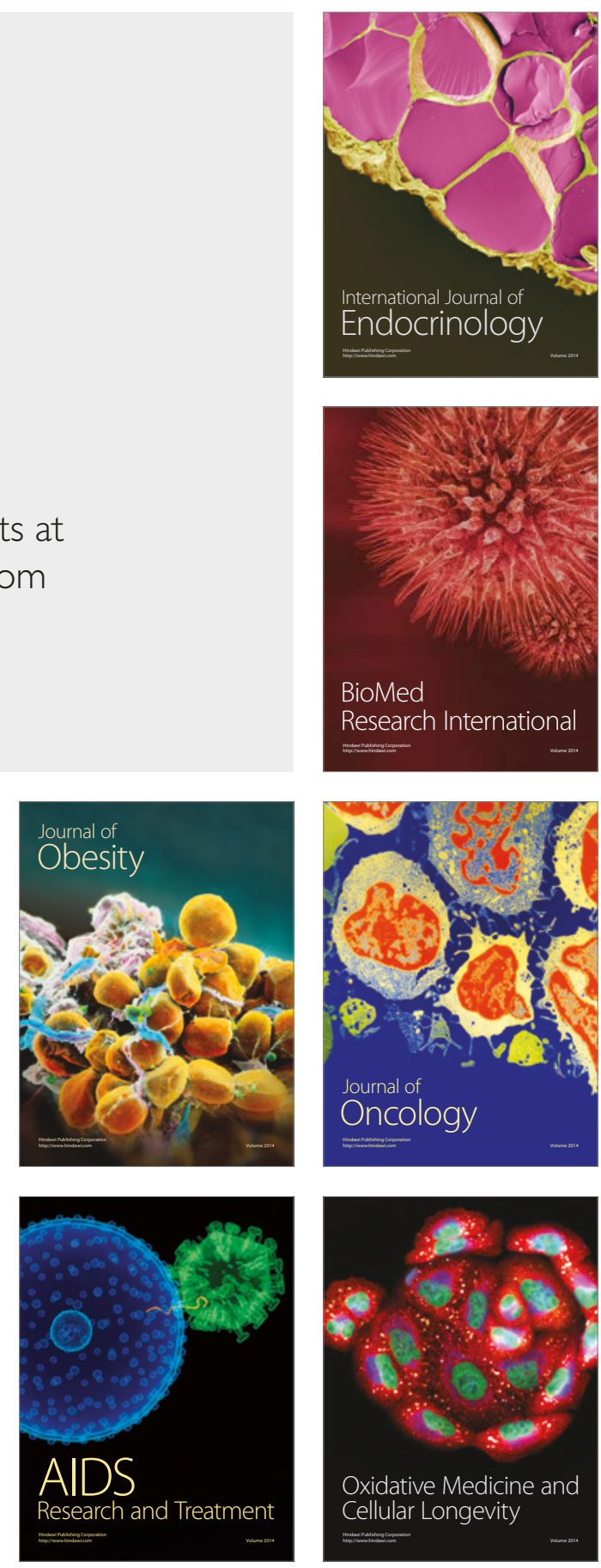